\title{
Fundamental parameters and origin of the very eccentric binary 41 Dra $^{\star}$
}

\author{
A. Tokovinin ${ }^{1}$, Y. Y. Balega ${ }^{2}$, E. A. Pluzhnik ${ }^{2}$, N. I. Shatsky ${ }^{3}$, N. A. Gorynya ${ }^{4}$, and G. Weigelt ${ }^{5}$ \\ 1 Cerro-Tololo Inter-American Observatory, Casilla 603, La Serena, Chile \\ 2 Special Astrophysical Observatory, Nizhnij Arkhyz, Zelenchuk region, Karachai-Cherkesia 369167, Russia \\ 3 Sternberg Astronomical Institute, 13 Universitetskii pr., 119992 Moscow, Russia \\ ${ }^{4}$ Institute of Astronomy of Russian Acad. Sci., 48 Pyatnitskaya St., 109017 Moscow, Russia \\ 5 Max-Planck-Institut für radioastronomie, Auf dem Hügel 69, 53121 Bonn, Germany
}

Received 17 March 2003 / Accepted 28 May 2003

\begin{abstract}
The evolutionary status and origin of the most eccentric known binary in a quadruple system, 41 Dra $(e=0.9754$, period $3.413 \mathrm{yr}$ ), are discussed. New observations include the much improved combined speckle-interferometric orbit, resolved photometry of the components and their spectroscopic analysis. The age of the system is $2.5 \pm 0.2 \mathrm{Gyr}$; all four components are likely coeval. The high eccentricity of the orbit together with known age and masses provide a constraint on the tidal circularization theory: it seems that the eccentric orbit survived because the convective zones of the F-type dwarfs were very thin. Now as the components of 41 Dra are leaving the Main Sequence, their increased interaction at each periastron passage may result in detectable changes in period and eccentricity.
\end{abstract}

Key words. stars: binaries: visual - stars: binaries: spectroscopic - stars: formation - stars: individual: HD 166866, HD 166865

\section{Introduction}

Multiple stars remain an active research topic, despite having been studied for more than 200 yrs. Nowadays the emphasis has shifted from determination of orbits and masses (although masses accurate to few percent are still needed to check stellar models) to investigation of specific and rare objects, to statistics of binary populations and to binary star formation. The multiple system studied here fits these criteria: it is a unique object with well-defined fundamental parameters that holds promise for elucidating some aspects of multiple-star formation.

Two 6th magnitude stars, 40 and 41 Dra (respectively, HR 6809 and 6810, HIP 88127 and 88136, HD 166865 and 166866), are 19" from each other on the sky and form a physical common proper motion couple $\Sigma 2308$ discovered by V. Struve in 1832 and also known as ADS 11061. Moreover, each is itself a spectroscopic binary, making the whole system quadruple. Hereafter we denote the visual components as $\mathrm{A}=41$ Dra and $\mathrm{B}=40$ Dra, and the spectroscopic components as $\mathrm{Aa}, \mathrm{Ab}, \mathrm{Ba}$, and $\mathrm{Bb}$.

The orbit of Aab published by Tokovinin (1995, hereafter T95) is very eccentric - the most eccentric among all known spectroscopic binaries. The Aab system was resolved by

Send offprint requests to: A. Tokovinin,

e-mail: atokovinin@ctio.noao.edu

* Tables 1,2, and 3 are only available in electronic form at the CDS via anonymous ftp to

cdsarc.u-strasbg.fr $(130.79 .128 .5)$ or via

http://cdsweb.u-strasbg.fr/cgi-bin/qcat?]/A+A/409/245 speckle interferometry (designated as BAG 6 in the WDS catalogue) and its preliminary interferometric orbit was computed by Balega et al. (1997). There are now enough speckle data to derive a new good-quality combined orbit. Radial velocities measured during the last passage through periastron in MayJune 2001 permit us to establish the orbital period very accurately and even to address the issue of orbit evolution.

The summary of interferometric measurements of the magnitude difference of Aab in different pass-bands, from visible to the infrared, is provided by Balega et al. (2001b) who show that $\Delta m$ is practically constant over a wide range of wavelengths, with the average value of $\Delta m=0.426 \pm 0.028$. It points to the fact that $\mathrm{Aa}$ and $\mathrm{Ab}$ have very similar effective temperatures and different radii. Low-resolution spectroscopy reported in that work was used to fit standard photospheric models in an attempt to find the best-matching effective temperatures and radii of the components $\mathrm{Aa}$ and $\mathrm{Ab}$. A similar analysis of both 40 and 41 Dra was done by Al Wardat (2002). There is some disagreement in the temperature estimates for 41 Dra (6500 and $6100 \mathrm{~K}$ ) between those two works, neither of which quotes errors on these numbers. High-resolution spectroscopy revealed that 41 Dra has a slight $(+0.2$ dex $)$ over-abundance of iron compared to the Sun (Balega et al. 2003). X-ray flux measured by Pizzolato et al. (2000) seems to be normal for F7V dwarfs.

The visual secondary component, 40 Dra, did not attract much attention, being a typical "garden variety" doublelined spectroscopic binary with $10.5 \mathrm{~d}$ period and moderately eccentric ( $e=0.38$ ) orbit which was first computed by 
Table 4. New combined orbit of 41 Dra.

\begin{tabular}{lcc}
\hline \hline Element & Value & Error \\
\hline Period $P$, days & 1246.680 & 0.004 \\
Periastron epoch $T, \mathrm{JD}$ & 2449571.037 & 0.008 \\
Eccentricity $e$ & 0.9754 & 0.0001 \\
Semi-major axis $a$, arcsec & 0.0706 & 0.0014 \\
Position angle of node $\Omega_{a}$, deg & 1.9 & 1.7 \\
Argument of periastron $\omega_{a}$, deg & 127.31 & 0.13 \\
Inclination $i$, deg & 49.7 & 2.9 \\
Primary amplitude $K_{1}, \mathrm{~km} \mathrm{~s}^{-1}$ & 44.62 & 0.10 \\
Secondary amplitude $K_{2}, \mathrm{~km} \mathrm{~s}^{-1}$ & 48.06 & 0.11 \\
System velocity $V_{0}, \mathrm{~km} \mathrm{~s}^{-1}$ & 5.76 & 0.05 \\
\hline Primary mass, $M_{\odot}$ & 1.28 & 0.15 \\
Secondary mass, $M_{\odot}$ & 1.20 & 0.14 \\
Orbital parallax, mas & 23.0 & 2.2 \\
\hline
\end{tabular}

Boothroyd (1922) and re-determined with greater accuracy in T95. Here we come back to this orbit to check whether its elements are stable over time.

\section{New combined orbit of 41 Dra}

The first double-lined spectroscopic orbit published in T95 was based on the periastron passage in August 1994 observed by N.I.S and N.A.G. The following periastron around January 1, 1998, was not covered because of cloudy sky. However, N.I.S and N.A.G did obtain the data on the next occasion, in May-June 2001. The observations were made with the 70-cm telescope at Moscow University campus using the correlation radial velocity meter (Tokovinin 1987). As in T95, the velocities were derived from fitting Gaussian curves to the correlation dips; partially blended dips were split by fixing the width and contrast of the fitted Gaussians. The individual velocities and their residuals to the new orbit are given in Table 1, published electronically. Table 2 lists the observations of the Bab binary obtained on this occasion.

All speckle interferometry of 41 Dra comes from the Russian $6 \mathrm{~m}$ telescope. We add to the published data another point taken in 2001.27 and provide in Table 3 all speckle measurements and their residuals.

Merging new and old data in the combined specklespectroscopic orbital solution yields the elements and their formal errors derived by weighted least-squares fitting (Table 4). Initially the weights were taken to be inversely proportional to the squares of observational errors, assumed to be 2 mas for speckle-interferometry. It turns out that speckle data are more precise, while the errors of radial velocities are larger than their formal estimates. The final solution was computed by equalizing the relative weights of interferometry and velocities, so that $\chi^{2} / N$ ratio is close to 1 for all kinds of data. The result did not change significantly compared to the initial weighting. The weighted rms residuals to the orbit are $0.53 \mathrm{~km} \mathrm{~s}^{-1}$ and $0.59 \mathrm{~km} \mathrm{~s}^{-1}$ for $\mathrm{Aa}$ and $\mathrm{Ab}$ velocities, $0.9^{\circ}$ and 1 mas for interferometry. One interferometric measurement (1994.71)

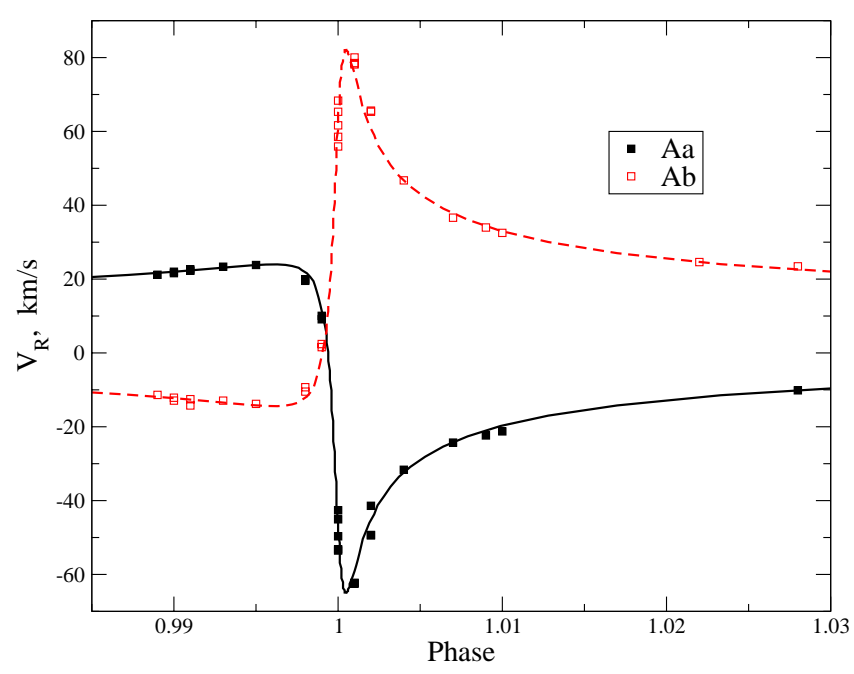

Fig. 1. Radial velocities of 41 Dra during the periastron passage in 2001. The primary component is denoted by filled squares and full line, secondary component by empty squares and dashed line. The descending portion of the Aa radial velocity curve lasts only 3 days.

with 25 mas separation - at the limit of $6 \mathrm{~m}$ telescope resolution - was excluded, but the overall quality of interferometry is excellent. This orbit represents the state-of-the-art in interferometry: a better orbit could be obtained only with longbaseline interferometers, but none of the current interferometers yet reaches 6-th magnitude stars. Note that two periastron observations define the $3.41 \mathrm{yr}$ period with an error of only 6 min., which might be the most accurate period among resolved binaries. The timing of periastron passages is accurate to $\sim 10 \mathrm{~min}$. because the component acceleration in this eccentric orbit is high (some $3 \mathrm{~km} \mathrm{~s}^{-1}$ per hour near periastron), despite the long period. In Fig. 1 we plot the radial velocities for the last periastron passage and in Fig. 2 we plot the interferometric observations.

The masses and orbital parallax are computed directly from the combined orbit. Estimates of their errors take into account correlations between the orbital elements. The mass ratio $q=M_{2} / M_{1}=0.928 \pm 0.003$ is determined with a much better accuracy than the masses themselves, hence any system model must precisely fit the mass ratio. The Hipparcos parallax (ESA 1997) was measured independently for A and B (18.8 \pm 1.8 and 19.6 \pm 3.8 mas respectively). It is manifestly wrong, being distorted by the orbital motion of Aab as noted by Shatskii \& Tokovinin (1998) for this and other similar systems.

The semi-major orbital axis is 3.1 AU The components approach each other at periastron to a distance of $0.078 \mathrm{AU}$ or $16.7 R_{\odot}$.

\section{Evolution of the orbital elements?}

New spectroscopic data on the systems of Aab and Bab obtained in 2001 can serve to study the possible evolution of those orbits.

We determined the eccentricity and semi-amplitudes of Aab from the 2001 velocities only, in the hope of detecting any change of orbital elements that might have occurred 


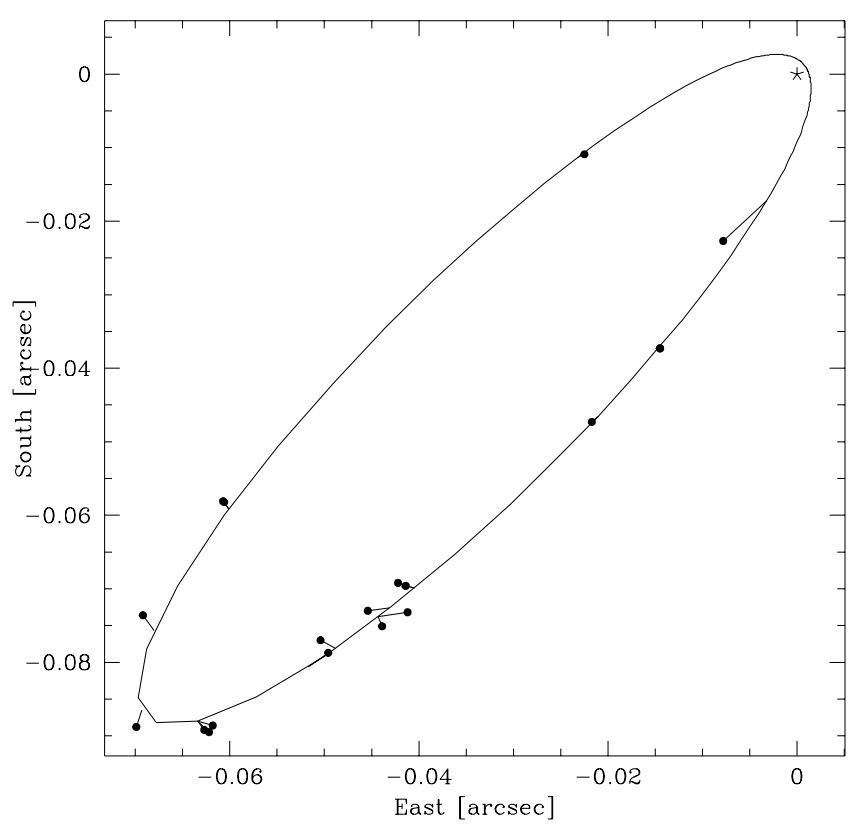

Fig. 2. Interferometric observations of 41 Dra (filled circles) are connected to the corresponding points on the orbit ellipse. The position of the primary is marked by the asterisk in the upper right corner.

Table 5. Orbital elements of 41 Dra in 1994 and 2001.

\begin{tabular}{lcccc}
\hline \hline Element & \multicolumn{2}{c}{1994} & \multicolumn{2}{c}{2001} \\
& Value & Error & Value & Error \\
\hline$T$, JD & 49571.047 & 0.009 & 52064.3929 & 0.0025 \\
$e$ & 0.9754 & 0.0001 & 0.9752 & 0.0001 \\
$\omega$, deg & 127.6 & 0.2 & 127.0 & 0.2 \\
$K_{1}, \mathrm{~km} \mathrm{~s}^{-1}$ & 44.79 & 0.14 & 44.72 & 0.13 \\
$K_{2}, \mathrm{~km} \mathrm{~s}^{-1}$ & 47.88 & 0.17 & 47.75 & 0.13 \\
$V_{0}, \mathrm{~km} \mathrm{~s}^{-1}$ & 5.84 & 0.05 & 5.75 & 0.09 \\
\hline
\end{tabular}

since the periastron passage in 1994. The result is presented in Table 5. Compared to 1994, the changes are below the significance level. But they are, curiously enough, going in the expected direction: the eccentricity is diminishing, accompanied by a decrease in both semi-amplitudes! Observations of further periastron passages will show if such fast eccentricity variation is indeed taking place.

A more sensitive test for orbit evolution is offered by period changes. If circularization proceeds with approximately constant periastron distance (Goldman \& Mazeh 1994), the quantity $P(1-e)^{3 / 2}$ is constant, so any change of eccentricity corresponds to the change of period, $\mathrm{d} P / P=1.5 \mathrm{~d} e /(1-e)$. Hence, if $e$ decreased by 0.0002 , the period would be shortened by $15 \mathrm{~d}$. Although $P$ is known to within $0.004 \mathrm{~d}$, we have not yet observed three periastron passages as needed to detect period variation. At this level of precision, an eccentricity change of $5 \times 10^{-8}$ is observable. Precise timing of future periastron passages is the best way to detect ongoing orbit circularization.

It was conjectured in T95 that the larger semi-amplitudes of Bab measured by Boothroyd in 1920 compared to the orbit in 1986-94 could result from the precession engendered by
Table 6. Orbital elements of 40 Dra in 1986-1994 and 2001.

\begin{tabular}{lcccc}
\hline \hline Element & \multicolumn{2}{c}{$1986-1994$} & \multicolumn{2}{c}{2001} \\
& Value & Error & Value & Error \\
\hline$P$, days & 10.52785 & 0.0020 & 10.52785 & $*$ \\
$T, \mathrm{JD}$ & 48000.008 & 0.011 & 52053.260 & 0.040 \\
$e$ & 0.374 & 0.003 & 0.380 & 0.008 \\
$\omega$, deg & 246.2 & 0.4 & 244.35 & 1.33 \\
$K_{1}, \mathrm{~km} \mathrm{~s}^{-1}$ & 39.14 & 0.14 & 39.01 & 0.39 \\
$K_{2}, \mathrm{~km} \mathrm{~s}^{-1}$ & 42.91 & 0.17 & 42.86 & 0.43 \\
$V_{0}, \mathrm{~km} \mathrm{~s}^{-1}$ & 5.76 & 0.07 & 5.31 & 0.19 \\
\hline
\end{tabular}

Table 7. Photometry of 41 and 40 Dra.

\begin{tabular}{llcccc}
\hline \hline Comp. & Source & $V$ & $B-V$ & $U-B$ & $V-R$ \\
\hline A & Tycho & 5.682 & 0.508 & - & - \\
& JM53 & 5.68 & 0.50 & -0.01 & - \\
& WBVR & 5.699 & 0.506 & - & 0.432 \\
B & Tycho & 6.022 & 0.513 & - & - \\
& JM53 & 6.04 & 0.51 & -0.01 & - \\
& WBVR & 6.066 & 0.507 & - & 0.435 \\
\hline
\end{tabular}

an invisible more distant companion, like in other such cases detected by Mayor \& Mazeh (1987). The 24 new observations of Bab obtained in 2001 were used to re-calculate the orbit. We adjusted all elements except the period (Table 6). The comparison with previous elements shows that there was no significant change, although both semi-amplitudes did decrease very slightly. The change in the systemic velocity $V_{0}$ is only marginal. If there is indeed a fifth component orbiting around $\mathrm{Bab}$, its period should be longer than $15 \mathrm{yrs}$ covered by our observations. It cannot be longer than $\sim 100 \mathrm{yr}$, otherwise this sub-system would be dynamically unstable against perturbations from A. Existence of the fifth companion seems unlikely.

\section{Physical parameters of the components}

We now know from the differential photometry of Aab that the colors of Aa and Ab are very similar. The combined colors of visual components $\mathrm{Aab}$ and Bab are also very close, despite the magnitude difference of $\Delta m=0.34$. Photometry from the Tycho space mission (ESA 1997), Johnson \& Morgan (1953, JM53) and Kornilov et al. (1991, WBVR) is summarized in Table 7.

Processing of the high signal-to-noise correlation dips measured in 2001 enabled us to find the equivalent widths of Aa and $\mathrm{Ab}$ dips as $1.20 \pm 0.01$ and $0.77 \pm 0.01 \mathrm{~km} \mathrm{~s}^{-1}$ and the projected rotation velocities $V \sin i$ of $5.7 \pm 0.3$ and $4.4 \pm$ $0.5 \mathrm{~km} \mathrm{~s}^{-1}$, confirming the T95 results. The estimates of magnitude differences $\Delta m$ were made in T95 under the assumption that all components are on the Main Sequence (MS). Now we know that the effective temperatures of all components are similar, which permits us to derive $\Delta m$ directly from the ratio of dip equivalent widths: $\Delta m=0.48 \pm 0.02$ for Aab (compare to 
Table 8. Physical parameters of the components.

\begin{tabular}{lccc}
\hline \hline Comp. & $M / M_{\odot}$ & $R / R_{\odot}$ & $M_{V}$ \\
\hline $\mathrm{Aa}$ & 1.39 & 1.93 & 2.99 \\
$\mathrm{Ab}$ & 1.30 & 1.63 & 3.42 \\
$\mathrm{Ba}$ & 1.32 & 1.57 & 3.28 \\
$\mathrm{Bb}$ & 1.20 & 1.32 & 3.85 \\
\hline
\end{tabular}

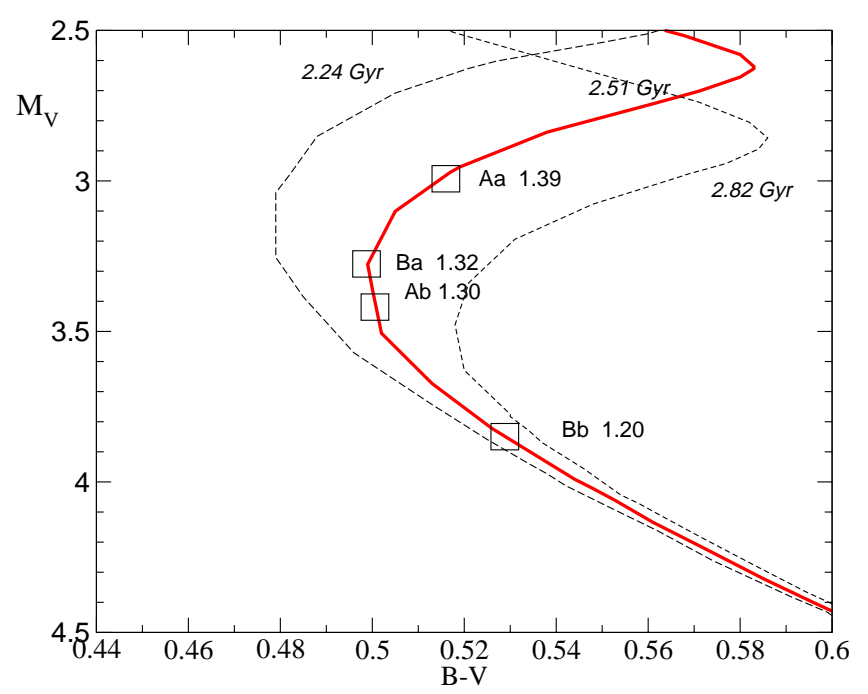

Fig. 3. Components of ADS 11061 on the isochrones of Girardi et al. (2000). The absolute magnitudes $M_{V}$ are measured, whereas the $B-V$ colors are "assigned" to match the combined colors and to make the components coeval. The numbers show component's masses.

$\Delta m=0.43$ from speckle-interferometry) and $\Delta m=0.56 \pm 0.03$ for Bab. This refers approximately to the photometric band $V$.

The orbital parallax corresponds to the distance modulus $m-M=3.19 \pm 0.25$ (refined to $m-M=3.25 \pm 0.05$ below). This distance and the photometry lead to the absolute magnitudes $M_{V}$ in the last column of Table 8 . Thus the observations constrain luminosities and colors of all 4 components to a narrow region of the Hertzsprung-Russel (H-R) diagram lying above the MS. The components are slightly evolved F-type stars; we show below that they can be coeval.

In Fig. 3 we place all 4 components on the evolutionary tracks computed by Girardi et al. (2000). We use the tracks for solar metallicity (cf. Balega et al. 2003) with over-shooting. Theoretical tracks are conveniently related to the observed values, $B-V$ and $M_{V}$, removing any additional uncertainty of bolometric corrections and effective temperature scale.

At the age of a few Gyr, as appropriate in this case, the stars evolve with fairly constant absolute magnitude $M_{V}$. This greatly simplifies our task: independently of the exact age, the mass of Aa component can be found directly from its $M_{V}$ as $1.39 M_{\odot}$. The mass of $\mathrm{Ab}$ follows from the measured mass ratio. These "evolutionary" masses of $\mathrm{Aa}$ and $\mathrm{Ab}$ are compatible with the directly measured masses. The same procedure is applied to $\mathrm{Ba}$ and $\mathrm{Bb}$, leading to the mass estimates given in Table 8. With the modeled mass sum of $\mathrm{Aa}+\mathrm{Ab} M=2.7 M_{\odot}$ we obtain parallax $\pi_{\text {dyn }}=a P^{-2 / 3} M^{-1 / 3}=22.4 \pm 0.5$ mas (here the semi-major axis $a$ is in arcseconds and period $P$ is in years).
Its error comes exclusively from the error of the orbital semimajor axis of Aab; it is the best current estimate of the distance to ADS 11061.

We do not know the individual $B-V$ color indices of the components with sufficient accuracy, but we can select an isochrone that matches the known combined colors of A and B. Such a fit is shown in Fig. 3. The $B-V$ colors of components were "assigned" to be on the isochrone. According to the model (Table 8), the combined $B-V$ colors of Aab and Bab are both equal to 0.51 , their $U-B$ colors are -0.01 . The modeled magnitude difference of the Aab pair is 0.39 in the $V$ band and 0.43 in the $K$ band. Selecting adjacent isochrones would lead to discrepant combined colors. So, the age of ADS 11061 can be established as $2.5 \pm 0.2$ Gyr. The effective temperatures of all components are similar, from the coolest $6280 \mathrm{~K}$ for $\mathrm{Bb}$ to the hottest $6380 \mathrm{~K}$ for $\mathrm{Ba}$. These estimates depend on the stellar models used.

Is the axial rotation of components affected by their close neighbors? The angular velocity of the Aa- $\mathrm{Ab}$ vector at periastron is $\left(1-e^{2}\right)^{1 / 2}(1-e)^{-2}=364$ times faster than the average orbital angular velocity. If Aa were synchronized at periastron, it would have the equatorial velocity of $28.2 \mathrm{~km} \mathrm{~s}^{-1}$, and $V \sin i=21.6 \mathrm{~km} \mathrm{~s}^{-1}$, assuming that rotational axes are perpendicular to the orbital plane and hence $i=50^{\circ}$. The measured $V \sin i\left(5.7\right.$ and $\left.4.4 \mathrm{~km} \mathrm{~s}^{-1}\right)$ show that both Aa and Ab rotate sub-synchronously. The inclination of the Bab system estimated from its spectroscopic mass sum $M \sin ^{3} i=0.273 M_{\odot}$ and the model mass sum $2.52 M_{\odot}$ is $35^{\circ}$. The synchronous rotational velocity is then expected to be $V \sin i=10.2 \mathrm{~km} \mathrm{~s}^{-1}$, to be compared with 7.2 and $6.7 \mathrm{~km} \mathrm{~s}^{-1}$ reported for $\mathrm{Ba}$ and $\mathrm{Bb}$ in T95. Hence, none of the four components is synchronized with the orbits.

\section{Orbit of the $A B$ system}

With the "evolutionary" mass sum 5.21 $M_{\odot}$ and the distance to the system $44.6 \mathrm{pc}$, we compute the orbit of AB by the dynamical method of Apparent Motion Parameters (AMP), as done previously by Kiselev \& Romanenko (1996) and T95. This method gives a family of orbits which depend on one unknown parameter, the distance between the components along the line of sight $r_{z}$. For elliptic orbits, the distance between the components $r$ obeys the inequality $r<r_{\max }=8 \pi^{2} M / V^{2}$, where the distance is in $\mathrm{AU}, V$ is in $\mathrm{AU} / \mathrm{yr}, M$ is in solar masses. The relative velocity $V$ of the components and their projected distance $r_{x y}$ are known and we can write $r_{z}=z \sqrt{r_{\max }^{2}-r_{x y}^{2}}$, so that $z= \pm 1$ corresponds to $r=r_{\max }$ (parabolic orbits). In Table 9 we list some of those orbits. Apart from new mass sum and distance, we used the same input data (relative position and proper motion) as in T95 and the velocity difference $V_{A}-V_{B}=0.05 \mathrm{~km} \mathrm{~s}^{-1}$ as follows from the spectroscopic orbits of Aab and Bab. Because of the short time base of the Hipparcos experiment, it measured the relative proper motion of AB with much less precision than ground-based photographic astrometry. The orbits in Table 9 sensitively depend on input data, e.g. on parallax, hence they should be considered as being only indicative of the possible orbital motion of $\mathrm{AB}$. 
Table 9. Orbits of the AB pair computed by the AMP method.

\begin{tabular}{cccccccc}
\hline \hline$z$ & $P$ & $e$ & $a$ & $\Omega_{B}$ & $\omega$ & $i$ & $\phi$ \\
& $\mathrm{yr}$ & & $\mathrm{AU}$ & ${ }^{\circ}$ & ${ }^{\circ}$ & ${ }^{\circ}$ \\
\hline-0.9 & 494060 & 0.88 & 10834 & 19 & 302 & 108 & 154 \\
-0.7 & 77030 & 0.71 & 3139 & 18 & 320 & 113 & 158 \\
-0.3 & 15171 & 0.75 & 1062 & 18 & 358 & 135 & 167 \\
0.0 & 10019 & 0.84 & 806 & 232 & 150 & 178 & 129 \\
0.3 & 15171 & 0.73 & 1062 & 200 & 174 & 133 & 85 \\
0.7 & 77030 & 0.69 & 3139 & 200 & 134 & 112 & 64 \\
0.9 & 494060 & 0.87 & 10834 & 199 & 117 & 108 & 60 \\
\hline
\end{tabular}

In the last column of Table 9 we give the relative angle $\phi$ between the angular momenta of the outer system $\mathrm{AB}$ and the inner system Aab computed as

$\cos \phi=\cos i_{\text {out }} \cos i_{\text {in }}+\sin i_{\text {out }} \sin i_{\text {in }} \cos \left(\Omega_{\text {out }}-\Omega_{\text {in }}\right)$.

It is interesting that all AMP orbits correspond to large relative inclinations between the orbits of $\mathrm{AB}$ and $\mathrm{Aab}$.

\section{Dynamics and origin of the multiple system}

In this section we try to understand why the orbit of Aab is so eccentric. First we evaluate the chances of orbit circularization to see how such high eccentricity could have survived over the life-time of this system. Then we investigate the dynamical origin of high eccentricity.

The very high eccentricity of the Aab orbit and its known age offer an interesting possibility to check theories of dissipative orbital circularization. Such a check was attempted for Gliese 586A (Goldman \& Mazeh 1994) with $e=0.9752$ and $P=890$ d. Less strict constraints were obtained from another eccentric binary, HD 2909, with $e=0.949, P=2128 \mathrm{~d}$ (Mazeh et al. 1995). In these works the semi-major axis evolution time $T_{a}$ is estimated as

$T_{a} \sim 5 \eta^{-1} T_{0}\left(P / P_{0}\right)^{16 / 3}(1-e)^{15 / 2}$,

where the constants $T_{0}=2 \times 10^{10} \mathrm{yr}, P_{0}=20 \mathrm{~d}$, and the viscosity constant $\eta=\left(\tau_{\mathrm{s}} / P_{0}\right)^{n}$ depends on the prescription as explained by Goldman \& Mazeh (1994): $n=0$ for unreduced viscosity, $n=1$ and $n=2$ for different theories. The convective time $\tau_{\mathrm{s}} \approx 0.27 \mathrm{~d}$ is estimated from the angular velocity at periastron, so $\eta=0.014^{n}$. Straightforward application to Aab gives $T_{a} \approx 3.3 \times 10^{8} \mathrm{yr}$ for $n=0$ and $T_{a} \approx 2.4 \times 10^{9} \mathrm{yr}$ for $n=1$.

The theory of tidal circularization is not yet free of uncertainties. The case of 41 and 40 Dra is particularly tricky because their components, while on the MS, were close to the border-line between convective and radiative stars. Even a small difference in mass has a large influence on the thickness of convective zones, especially for Aa and Ab. We speculate that the orbit of Bab is more circular (though still not quite so, $e=0.38$ ) because $\mathrm{Ba}$ and $\mathrm{Bb}$ have lower masses and thicker convective zones than $\mathrm{Aa}$ and $\mathrm{Ab}$, whereas periastron distances in both sub-systems are equal. In short, it seems plausible that the highly-eccentric orbit of Aab escaped tidal circularization, and the still eccentric orbit of Bab confirms this hypothesis. Sub-synchronous rotation is an additional symptom of the inefficiency of tidal friction in Aab and Bab.

If tidal orbit evolution preserves the periastron distance, the final orbital periods of Aab and Bab after complete circularization can be computed as $P(1-e)^{3 / 2}$. They turn out to be remarkably similar, 4.8 and 5.1 days respectively. Such periods are very frequent among spectroscopic sub-systems in late-type multiple stars (Tokovinin \& Smekhov 2002).

Multiple systems can be chaotic or hierarchical; for ADS 11061 either may be true, with hierarchical configurations being more probable (T95). If ADS 11061 were chaotic, we must admit that it survived over $\sim 10^{6}$ crossing times, a very unlikely hypothesis. So we believe that this multiple system is hierarchical and dynamically stable. The ratio of the periastron distance of $\mathrm{AB}$ to the semi-major axis of Aab $a_{\text {out }}\left(1-e_{\text {out }}\right) / a_{\text {in }}$ that follows from Table 9 is over 40, confirming this statement.

The high eccentricity of an inner orbit in a hierarchical multiple system can be attained by the Kozai mechanism (Kozai 1962). If the inner orbit were initially almost perpendicular to the orbital plane of the outer system, it will change its inclination and eccentricity periodically while preserving the Kozai invariant $\Theta=\left(1-e^{2}\right) \cos ^{2} \phi$, where $\phi$ is the angle between the inner and outer orbital angular momentum vectors. The period of the Kozai cycle is estimated as

$T_{\text {Kozai }} \sim P_{\text {out }}^{2} / P_{\text {in }}\left(1-e_{\text {out }}\right)^{3 / 2}$.

Kozai cycles are perturbed by the apsidal rotation in the inner system caused by a component's structure and relativistic precession. The period of relativistic precession is

$T_{\text {rel }}=3.36 \times 10^{7}\left(1-e_{\text {in }}^{2}\right) P a / M \mathrm{yr}$,

where $P$ is in years, $a$ in AU, mass $M$ in solar masses (Holman et al. 1997). For the Aab system $T_{\text {rel }}=6 \mathrm{Myr}$. If $T_{\text {rel }}<T_{\text {Kozai }}$, Kozai cycles can no longer occur. Given that $T_{\text {rel }}$ depends on $e_{\text {in }}$ and $T_{\text {Kozai }}$ does not, the condition $T_{\text {rel }} \sim T_{\text {Kozai }}$ will be reached if $e_{\text {in }}$ grows sufficiently during the first Kozai cycle. When this happens, the relativistic rotation of the line of apsides averages out the Kozai effect, so $e_{\text {in }}$ will not evolve periodically and will remain high. This consideration shows that very similar periastron distances in Aab and Bab may be not a coincidence but rather a consequence of the interplay between Newtonian and relativistic dynamics in this multiple system.

Now we estimate $T_{\text {Kozai }}$. The outer period $P_{\text {out }}$ is quite uncertain, however. The values of $z=0,0.3,0.7$ from Table 9 correspond to $T_{\text {Kozai }}$ of $1.9,10$, and $300 \mathrm{Myr}$, respectively. So, the condition $T_{\text {Kozai }} \approx T_{\text {rel }}$ can be satisfied for some plausible elements of the $\mathrm{AB}$ orbit. It seems thus likely that the high eccentricity of Aab is indeed a result of the dynamical evolution outlined above which occurred in the first $10 \mathrm{Myr}$ after formation of the multiple system.

The most likely evolutionary scenario of ADS 11061 is as follows (Fig. 4). This quadruple system was formed $2.5 \mathrm{Gyr}$ ago from a small gas cloud. Initially, the orbits of Aab and Bab had long periods, were not very eccentric and almost perpendicular to the orbit of the outer system AB. Within $\sim 10 \mathrm{Myr}$ 


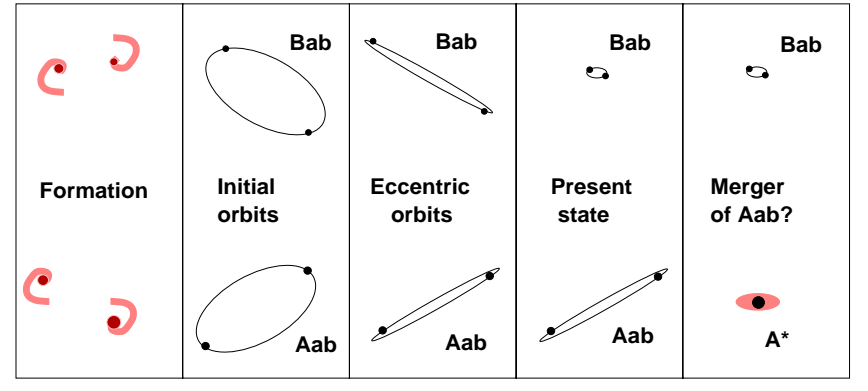

Fig. 4. Possible evolution of the ADS 11061 multiple system.

both inner orbits acquired high eccentricity, the latter being limited by the relativistic precession. During the following 2.5 Gyr the Bab sub-system was partially circularized, decreasing its period to $10.5 \mathrm{~d}$, while the circularization of Aab did not occur because its components had thinner convective zones. If both sub-systems were less massive, they would have been completely circularized now with orbital periods around $5 \mathrm{~d}$.

Presently the components of Aab increase their radii, evolving off the MS. Their strong interaction at each periastron leads to significant orbital circularization which may even be detectable by a careful timing of future periastron passages. Further expansion of $\mathrm{Aa}$ and $\mathrm{Ab}$ may result in a merger, leaving an unusual triple with apparently non-coeval components until Bab follows the same route and only a wide pair of blue stragglers remains.

\section{Conclusions}

The scenario outlined above can be checked with modern tools for joint modeling of nuclear and dynamical stellar evolution. New constraints on the still obscure physics of tidal interaction may be obtained from this system when it is compared to models.

On the observational side, 40 and 41 Dra will be excellent candidates for long-baseline interferometers when they are able to observe $6^{m}$ stars. Improved accuracy of the Aab orbit will lead to very precise mass and distance determination.
By resolving $\mathrm{Bab}$, it will be possible to determine the inclination of its orbit relative to $\mathrm{AB}$ and $\mathrm{Aab}$, thus achieving a complete dynamical description of this interesting quadruple system.

Acknowledgements. We thank R. Zhuchkov for a careful reading of the manuscript and for pointing out some errors. The comments of the anonymous referee helped to improve the article.

\section{References}

Al Wardat, M. A. 2002, Bull. Spec. Astrophys. Obs., 53, 51

Balega, Y. Y., Leushin, V. V., \& Weigelt, G. 2003, Bull. SAO, 55, in press

Balega, I. I., Balega, Y. Y., Hofman, K.-H., et al. 2002, A\&A, 385, 87

Balega, Yu. Yu., Leushin, V. V., \& Pluzhnik, E. A. 2001b, Bull. Spec. Astrophys. Obs., 51, 61

Balega, I. I., Balega, Y. Y., Hofman, K.-H., \& Weigelt, G. P. 2001a, Astron. Lett., 27, 95

Balega, I. I., Balega, Y. Y., Maksimov, A. F., et al. 1999, A\&AS, 140, 287

Balega, I. I., Balega, Y. Y., Falcke, H., et al. 1997, Astron. Lett., 23, 172

Boothroyd, S. L. 1922, Publ. Dom. Astrophys. Obs., 1, 246

ESA 1997, The Hypparcos and Tycho Catalogues. ESA SP-1200

Girardi, L., Bressan, A., Bertelli, G., \& Chiosi, C. 2000, A\&AS, 141, 371

Goldman, I., \& Mazeh, T. 1994, ApJ, 429, 362

Holman, M., Touma, J., \& Tremaine, S. 1997, Nature, 386, 254

Johnson, H. L., \& Morgan, W. W. 1953, ApJ, 117, 313 (JM53)

Kiselev, A. A., \& Romanenko, L. G. 1996, Astron. Rep., 40, 795

Kornilov, V. G., Volkov, I. M., Zakharov, A. I., et al. 1991, Catalog of WBVR magnitudes of Bright Northern-Sky Stars. Trudy Astron. Inst. Sternberg (Moscow: Moscow State University), 63

Kozai, Y. 1962, AJ, 67, 591

Mayor, M., \& Mazeh, T. 1987, A\&A, 171, 157

Mazeh, T., Zucker, S., Goldberg, D., et al. 1995, ApJ, 449, 909

Pizzolato, N., Maggio, A., \& Sciortino, S. 2000, A\&A, 361, 614

Shatskii, N. I., \& Tokovinin, A. A. 1998, Astron. Lett., 24, 673

Tokovinin, A. A. 1987, Sov. Astron., 31, 98

Tokovinin, A. A. 1995, Astron. Lett., 21, 250 (T95)

Tokovinin, A. A., \& Smekhov, M. G. 2002, A\&A, 382, 118 